\title{
ICU rehabilitation is associated with reduced long-term mortality from sepsis in patients with low skeletal muscle mass: a case control study
}

\author{
Taehwa Kim ${ }^{1}$, Sungchul Huh ${ }^{2}$, Soo-Yeon Kim ${ }^{2}$, Junhee Han ${ }^{3}$, Seung Eun Lee ${ }^{1}$, Woo Hyun Cho ${ }^{1}$, \\ Yun Seong Kim ${ }^{1}$, Doo Soo Jeon ${ }^{1}$, Hye Ju Yeo ${ }^{1}$ \\ ${ }^{1}$ Division of Pulmonology, Allergy and Critical Care Medicine, Department of Internal Medicine, ${ }^{2}$ Department of Rehabilitation Medicine, Pusan \\ National University Yangsan Hospital, Yangsan, Republic of Korea; ${ }^{3}$ Department of Statistics, Hallym University, Chuncheon, Korea \\ Contributions: (I) Conception and design: HJ Yeo; (II) Administrative support: S Huh, YS Kim; (III) Provision of study materials or patients: SE \\ Lee, WH Cho, T Kim; (IV) Collection and assembly of data: DS Jeon YS Kim; (V) Data analysis and interpretation: J Han, HJ Yeo, T Kim; (VI) \\ Manuscript writing: All authors; (VII) Final approval of manuscript: All authors. \\ Correspondence to: Hye Ju Yeo, MD, PhD. Division of Pulmonology, Allergy and Critical Care Medicine, Department of Internal Medicine, Pusan \\ National University Yangsan Hospital, Geumo-ro 20, Beomeo-ri, Mulgeum-eup, Yangsan-si, Gyeongsangnam-do 626-770, Republic of Korea. \\ Email: hjyeo@pusan.ac.kr.
}

Background: It is unclear whether intensive care unit (ICU)-rehabilitation reduces mortality from sepsis in low skeletal muscle mass. We evaluated whether the association of ICU-rehabilitation with mortality from sepsis differs between patients with and without low skeletal muscle mass.

Methods: We retrospectively reviewed 516 patients with sepsis who were admitted to the ICU between June 2011 and August 2017. The skeletal muscle area at the level of the third lumbar vertebra was measured with CT on admission. Patients were divided into two groups (low skeletal muscle mass and non-low skeletal muscle mass), and clinical outcomes were compared in patients treated with ICU-rehabilitation and without ICU-rehabilitation within each subgroup. We used Cox regression to examine factors associated with 1-year mortality in each subgroup.

Results: Low skeletal muscle mass was diagnosed in 421 (81.6\%). ICU-rehabilitation was conducted to $51.1 \%$ low skeletal muscle mass patients and 54.7\% non-low skeletal muscle mass patients. In the low skeletal muscle mass subgroup, in-hospital mortality $(26.0 \%$ vs. $39.8 \%, \mathrm{P}=0.003)$ and 6 -month mortality $(38.6 \%$ vs. $51.5 \%, \mathrm{P}=0.008)$ were lower in the ICU-rehabilitation group. However, there were no differences in the non-low skeletal muscle mass group. In the multivariate analysis, ICU-rehabilitation was independently associated with reduced 1-year mortality in low skeletal muscle mass patients (HR: 0.66, 95\% CI: 0.49-0.87, $\mathrm{P}=0.003$ ), but not in non-low skeletal muscle mass patients.

Conclusions: ICU-rehabilitation was independently associated with reduced 1-year mortality from sepsis among low skeletal muscle mass patients, but not among non-low skeletal muscle mass patients. Therefore, the delayed initiation of ICU-rehabilitation should be avoided, especially in low skeletal muscle mass patients.

Keywords: Low skeletal muscle mass; sepsis; ICU-rehabilitation; mortality

Submitted Jul 05, 2019. Accepted for publication Aug 21, 2019.

doi: 10.21037/atm.2019.08.117

View this article at: http://dx.doi.org/10.21037/atm.2019.08.117 


\section{Introduction}

Owing to recent advances in critical care, the early-mortality rate observed in sepsis has decreased, but many patients are still unable to return home (1-3). Many survivors are exposed to post-intensive care syndrome, such as intensive care unit (ICU)-acquired weakness and delirium, which leads to readmission and late-mortality (4). Notably, muscle wasting is seen in $50-100 \%$ of septic patients in ICU and has adverse impacts on clinical outcomes, including quality of life, as well as short-term and long-term prognosis $(5,6)$. The mechanisms of muscle wasting in sepsis are complex and multifactorial (7). Systemic inflammation, immobility, and multiple organ failure lead to muscle weakness, loss of muscle mass and ICU acquired weakness (8). Furthermore, these detrimental effects might be more apparent in patients with lower basal muscle mass.

Rehabilitation has recently emerged as an important field in critical care (9). Rehabilitation has anti-inflammatory effects, with the potential to reduce local cytokine expression and increase the expression of anti-apoptotic factors (10). Furthermore, it is important for the prevention and treatment of muscle wasting (11). In particular, early rehabilitation in ICU has been reported to improve clinical outcomes, such as decreasing the duration of mechanical ventilation and hospital length-of-stay and improving functional disability (9). Although recent guidelines recommend ICU-rehabilitation for critically ill patients, it is unclear whether ICU-rehabilitation improves mortality in patients with sepsis (12-14). Previous studies have focused on the hazardous effects of low skeletal muscle mass in sepsis. Generally, the clinical effect of rehabilitation differs according to the basal muscle status in sepsis (15). However, the effect of rehabilitation in patients with different amounts of basal muscle mass has not been evaluated in sepsis until now. In this study, we evaluated whether low skeletal muscle mass and ICU-rehabilitation impact clinical outcomes of sepsis, and whether ICU rehabilitation could improve mortality in septic patients with low skeletal muscle mass.

\section{Methods}

Rehabilitation for ICU patients is an approved health care service covered by the Korean Ministry of Health, Labor, and Welfare. Therefore, it would have been unethical to include a non-rehabilitation control group in this study. Therefore, we performed a retrospective case control study to evaluate the impact of low skeletal muscle mass and ICU-rehabilitation on mortality in sepsis. This study was approved by the institutional review board at our center (Pusan National University Yangsan Hospital, No. $05-$ 2018-082). The need for informed consent was waived because of the retrospective nature of the study, which used medical records maintained during routine care. We reviewed medical records of all adults with sepsis treated at our center between June 2011 and August 2017. Sepsis was defined according to the new definition (16). Patients who did not receive an abdominal computed tomography (CT) scan within $24 \mathrm{~h}$ of ICU admission, patients with scoliosis, and patients who died within $48 \mathrm{~h}$ of ICU admission were excluded. Generally, early death ( $\leq 48 \mathrm{~h}$ ) is mainly affected by uncontrollable organ failure, and such patients are unable to start rehabilitation. Therefore, we did not evaluate the effectiveness of ICU-rehabilitation in cases of early death.

Of the 940 patients with sepsis ( $\geq 18$ years), 516 patients were included in this study (Figure 1). We collected baseline data such as age, sex, body mass index (BMI), source of infection, serum lactate level on admission, presence of septic shock, Acute Physiology and Chronic Health Evaluation (APACHE) II score, Sequential Organ Failure Assessment (SOFA) score on admission to ICU, Charlson Comorbidity Index (CCI), length of hospital stay, hospital mortality, and 6-month, 1-year mortality.

\section{Measurement of skeletal muscle index (SMI)}

In this study, low skeletal muscle mass was assessed using an abdominal CT scan performed within $24 \mathrm{~h}$ of ICU admission. We calculated the psoas muscle area $\left(\mathrm{cm}^{2}\right)$ at the level of the third-lumbar vertebra, which is related to the total skeletal muscle mass. The psoas cross-sectional area was quantified within the range of -29 to +150 Hounsfield units using Slice-O-Matic version 5.0 (Tomovision; Magog, QC, Canada). The values for the left and right sides were summed. Measurements were divided by the patient's height in meters squared and expressed as the psoas SMI. CT scan analyses were performed by three trained researchers. Low skeletal muscle mass was defined as SMI $<55 \mathrm{~cm}^{2} / \mathrm{m}^{2}$ for males and $<39 \mathrm{~cm}^{2} / \mathrm{m}^{2}$ for females (17).

\section{Rebabilitation regimen}

In our hospital, rehabilitation in the ICU has been provided since March 2014. Each patient's rehabilitation regimen was individually developed and initiated by a multidisciplinary 


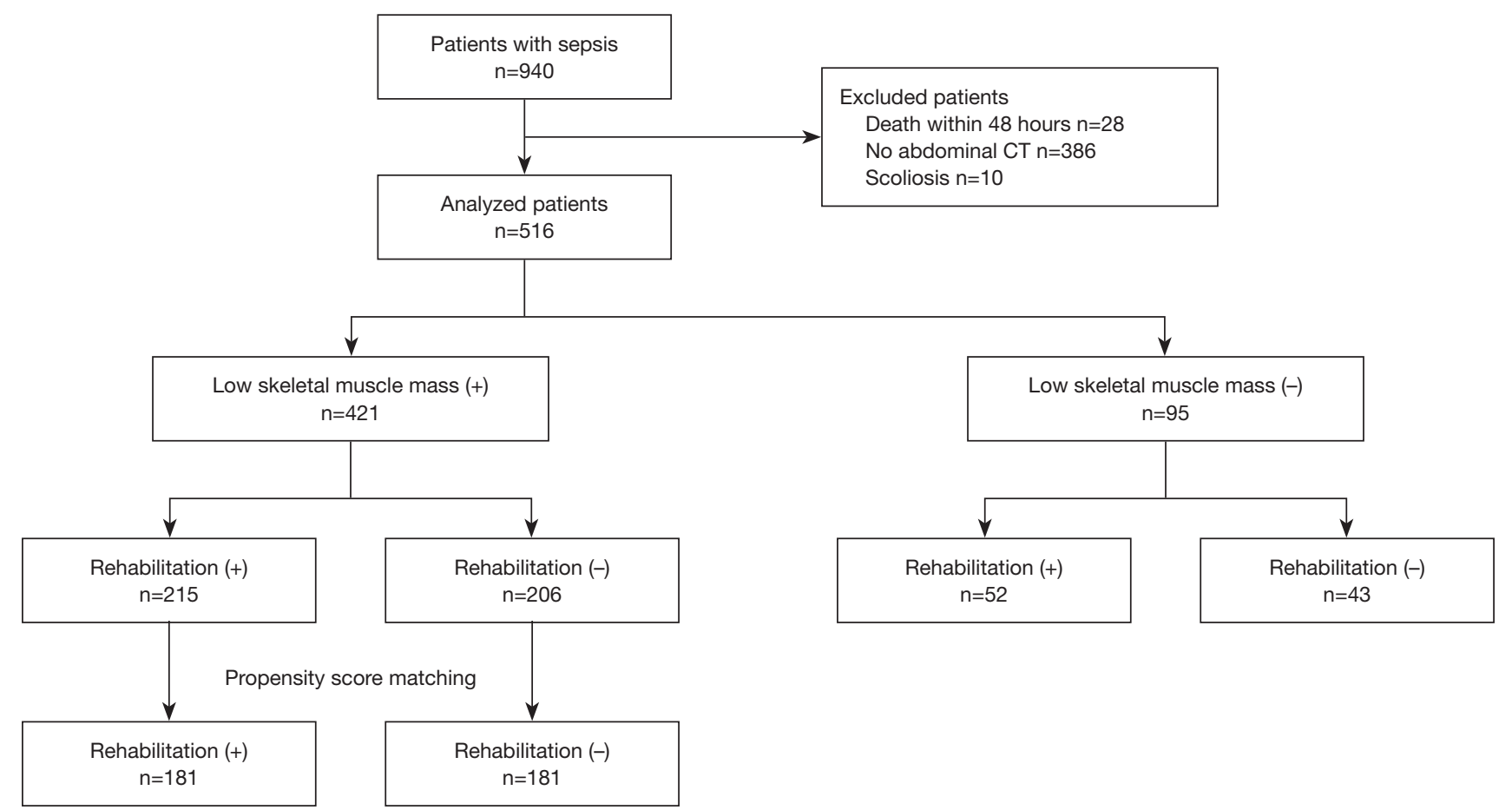

Figure 1 Chart depicting the flow of patients through the study. During the study period, 940 patients with sepsis were admitted to our hospital. Of these patients, 516 were included in the present study. +, presence; -, absence.

team that included a rehabilitation doctor, an intensivist, a physical therapist, and/or an occupational therapist, depending on the condition of each patient. The members of the rehabilitation team modified each patient's activity level in the ICU, changed the sedation practice from continuous infusion to as-needed boluses, provided both physical therapy and occupational therapy, and implemented safety screening for rehabilitation in the ICU. In general, rehabilitation was initiated after hemodynamic stabilization, with or without vasopressor use. To maintain physical ability and prevent the development of low skeletal muscle mass during ICU treatment, patients were treated through exercise therapy, as instructed by physical therapists. The patients were classified into four groups according to the severity of illness and their sedation level. Rehabilitation for the patients consisted of stretching, strengthening exercises and neuromuscular electrical stimulation to prevent muscle wasting. Level 1 included unconscious patients who could not participate in active rehabilitation. For those patients, only passive range of motion exercises for the upper and lower extremity joints were allowed. Level 2 included conscious patients with upper and lower extremity motor function grades that were less than fair. Both assistive active range of motion exercises with sitting training were allowed. The Level 3 group included conscious patients with upper extremity motor function grades that were greater than fair. These patients went through dangling with active range of motion exercise for all joints. Level 4 patients had upper and lower extremity motor function grades that were greater than fair. These patients performed dangling and standing exercises with assistance, as well as active range of motion exercises. Rehabilitation in the ICU was performed for 30 minutes every day. Care was taken to avoid disturbing cannulas, catheters, and ventilator lines. Patients with swallowing dysfunction were fed via a nasogastric tube, and those without swallowing dysfunction were fed orally. Dysphagia therapy was carried out for conscious patients with swallowing difficulty.

\section{Nutrition therapy}

ICU patients received nutritional care according to the documented protocol. Details include an energy requirement of $25-35 \mathrm{kcal} / \mathrm{kg}$ ideal body weight/day, protein intake of $1.2-2.0 \mathrm{~g} / \mathrm{kg} /$ day (if patients had no protein intolerance), and fat intake with lipid: energy ratio of $25 \%$. 


\section{Statistical analyses}

Continuous variables are expressed as mean \pm standard deviation. Categorical variables are expressed as numbers and percentages. To investigate the impact of low skeletal muscle mass on clinical outcomes in sepsis, baseline characteristics and outcomes were compared between two groups using a $t$-test or Fisher's exact test, where appropriate. Multivariate Cox regression analyses with backward stepwise selection method were performed to identify the independent clinical factors for 1-year mortality in sepsis; $\mathrm{P}$ values $<0.05$ were considered significant. Odds ratios (OR) are reported with corresponding $95 \%$ confidence intervals (CI). Furthermore, subgroup analysis was performed to evaluate the effects of ICU rehabilitation on mortality in septic patients with low skeletal muscle mass. We evaluated the clinical factors associated with 1 -year mortality in this subgroup. To approximate a random assignment, the propensity score matching method (1:1) was used to create sets of low skeletal muscle mass patient groups with and without rehabilitation specifically, which were adjusted for their ages and CCI. Survival curves and rates were obtained by Kaplan-Meier analyses, and group differences in survival were compared using the log-rank test. All statistical analyses were performed using R software (ver. 3.0; R Development Core Team, Vienna, Austria).

\section{Results}

\section{Patient characteristics and clinical outcomes according to basal muscle mass}

During the study period, 940 patients with sepsis were admitted to our hospital. Of these patients, 516 were included in the present study (Figure 1). Low skeletal muscle mass was diagnosed in $421(81.6 \%)$ patients. Baseline clinical characteristics and outcomes for all subjects are shown in Table 1. The mean age was higher in the low skeletal muscle mass group compared to the non-low skeletal muscle mass group (68.7 vs. 64.8 years, $\mathrm{P}=0.020$ ). The mean BMI and SMI were lower in the low skeletal muscle mass group compared to the non-low skeletal muscle mass group (BMI: 21.1 vs. $23.7 \mathrm{~kg} / \mathrm{m}^{2}, \mathrm{P}<0.001$; SMI: 33.4 vs. $\left.57.5 \mathrm{~cm}^{2} / \mathrm{m}^{2}, \mathrm{P}<0.001\right)$. ICU-rehabilitation was conducted in $215(51.1 \%)$ of the low skeletal muscle mass patients and 52 (54.7\%) of the non-low skeletal muscle mass patients. Although there was no difference in hospital mortality between the two groups, 6-month and 1-year mortality were both higher in the low skeletal muscle mass group compared to the non-low skeletal muscle mass group (6-month mortality: $44.9 \%$ vs. $26.3 \%, \mathrm{P}=0.001$; 1 -year mortality: $50.1 \%$ vs. $32.6 \%, \mathrm{P}=0.002)$. As well, the rate of discharge to home was lower in the low skeletal muscle mass group compared to the other $(39.4 \%$ vs. $58.9 \%, \mathrm{P}=0.001)$.

\section{Cox regression analysis for 1-year mortality}

Univariate analyses showed age (OR: 1.03, 95\% CI: 1.021.04, $\mathrm{P}<0.001)$, renal replacement therapy (RRT) (OR: 2.14, 95\% CI: $1.66-2.77, \mathrm{P}<0.001)$, multiple vasopressor use (OR: 2.33, 95\% CI: 1.58-3.43, $\mathrm{P}<0.001)$, CCI (OR: 1.14, 95\% CI: $1.09-1.20, \mathrm{P}<0.001$ ), SOFA (OR: 1.07, 95\% CI: 1.04-1.10, $\mathrm{P}<0.001$ ), low skeletal muscle mass (OR: 1.71, 95\% CI: 1.17-2.49, $\mathrm{P}=0.006)$, and ICU rehabilitation (OR: 0.70, 95\% CI: 0.54-0.90, $\mathrm{P}=0.005$ ) were associated with 1-year mortality (Table 2). All of these variables were included in the multivariate analysis, which showed age (OR: 1.02, 95\% CI: $1.00-1.03, \mathrm{P}=0.008)$, RRT (OR: $2.09,95 \%$ CI: 1.60-2.73, $\mathrm{P}<0.001)$, multiple vasopressor use (OR: 2.14 , 95\% CI: 1.43-3.20, $\mathrm{P}<0.001$ ), CCI (OR: 1.09, 95\% CI: 1.02-1.17, $\mathrm{P}=0.009$ ), low skeletal muscle mass (OR: 1.47, 95\% CI: $1.01-2.16, \mathrm{P}=0.046)$ and rehabilitation (OR: 0.65, 95\% CI: $0.50-0.85, \mathrm{P}=0.002)$ were associated with 1 -year mortality.

\section{Clinical characteristics of the low skeletal muscle mass group according to ICU-rehabilitation}

The baseline characteristics, depending on the ICUrehabilitation status, of the low skeletal muscle mass patients are shown in Table 3. The mean age and BMI were lower in the rehabilitation group compared to the nonrehabilitation group (age: 66.8 vs. 70.7 years, $\mathrm{P}=0.005$; BMI: 20.6 vs. $21.7 \mathrm{~kg} / \mathrm{m}^{2}, \mathrm{P}=0.006$ ). The incidence of urinary tract infection was higher in the rehabilitation group compared to the other $(15.3 \%$ vs. $7.8 \%, \mathrm{P}=0.015)$. The mean hospital length-of-stay $(73.2$ vs. 35.5 days, $\mathrm{P}<0.001)$ and ICU length-of-stay (22.5 vs. 15.9 days, $\mathrm{P}=0.004)$ were higher in the rehabilitation group. However, hospital mortality (26\% vs. 39.8\%, $\mathrm{P}=0.003)$ and 6-month mortality (38.6\% vs. $51.5 \%, \mathrm{P}=0.008)$ were lower in the rehabilitation group compared to the non-rehabilitation group. As well, the rate of discharge to home was higher in the rehabilitation group compared to the non-rehabilitation group ( $43.3 \%$ vs. $35.4 \%, \mathrm{P}=0.011)$. 
Table 1 Basal characteristics and outcomes of patients with and without low skeletal muscle mass

\begin{tabular}{|c|c|c|c|}
\hline Variable & Non-low skeletal muscle mass ( $\mathrm{N}=95)$ & Low skeletal muscle mass ( $\mathrm{N}=421)$ & $\mathrm{P}$ \\
\hline Male & $54(56.8)$ & $289(68.6)$ & 0.028 \\
\hline $\mathrm{BMI}\left(\mathrm{kg} / \mathrm{m}^{2}\right)$ & $23.7 \pm 3.3$ & $21.1 \pm 4.1$ & $<0.001$ \\
\hline $\mathrm{SMI}\left(\mathrm{cm}^{2} / \mathrm{m}^{2}\right)$ & $57.5 \pm 15.1$ & $33.4 \pm 10.1$ & $<0.001$ \\
\hline SOFA & $8.2 \pm 4.4$ & $8.2 \pm 4.0$ & 0.999 \\
\hline $\mathrm{CCl}$ & $4.2 \pm 2.5$ & $4.3 \pm 2.2$ & 0.808 \\
\hline Multiple vasopressor use & $71(74.7)$ & $339(80.5)$ & 0.207 \\
\hline Renal replacement therapy & $28(29.5)$ & $126(29.9)$ & 0.903 \\
\hline Respiratory & $81(85.3)$ & $373(88.6)$ & 0.366 \\
\hline Abdominal & $9(9.5)$ & $30(7.1)$ & 0.434 \\
\hline Urinary tract & $14(14.7)$ & $49(11.6)$ & 0.405 \\
\hline Soft tissue & $8(8.4)$ & $20(4.8)$ & 0.154 \\
\hline Rehabilitation & $52(54.7)$ & $215(51.1)$ & 0.518 \\
\hline Ventilator day & $26.6 \pm 34.1$ & $24.9 \pm 39.6$ & 0.772 \\
\hline Hospital stay & $44.4 \pm 45.4$ & $54.8 \pm 63.0$ & 0.065 \\
\hline ICU stay & $17.4 \pm 23.4$ & $19.3 \pm 23.9$ & 0.492 \\
\hline
\end{tabular}

Values are expressed as mean \pm standard deviation or $\mathrm{n}(\%)$. BMI, body mass index; SMI, skeletal muscle index; APACHE II, Acute Physiology and Chronic Health Evaluation II; SOFA, Sequential Organ Failure Assessment; CCl, Charlson Comorbidity Index.

\section{Cox regression \& Kaplan-Meier analysis for 1-year mortality in the low skeletal muscle mass group}

Results of the multivariate Cox regression analysis to identify factors associated with 1-year mortality are shown in Table 4. ICU-rehabilitation (OR: 0.66, 95\% CI: 0.49-0.87, $\mathrm{P}=0.003$ ), RRT (OR: 1.96, 95\% CI: 0.47-2.61, $\mathrm{P}<0.001$ ), multiple vasopressor use (OR: 1.92, 95\% $\mathrm{CI}$ : $1.26-2.92, \mathrm{P}=0.002$ ) and CCI (OR: 1.08, 95\% CI: 1.01-1.16, $\mathrm{P}=0.033$ ) were significantly associated with 1 -year mortality. The KaplanMeier survival curve showed low skeletal muscle mass patients who did not receive ICU rehabilitation had significantly higher 1 -year mortality $\left(\chi^{2}=7.35, \mathrm{P}=0.007\right.$, Figure S1).

\section{Propensity score matching analysis in the low skeletal muscle mass group}

To adjust for age, BMI, and infection source in low skeletal muscle mass patients, the propensity score matching method (1:1) was used to create sets of low skeletal muscle mass patient groups with and without rehabilitation. After propensity score matching, the final analyses included 362 patients, composed of a rehabilitation group $(n=181)$ and non-rehabilitation group $(n=181)$. There were no significant differences in baseline characteristics between the two groups. The clinical outcomes following propensity score matching are presented in Figure 2. Mean hospital 
Table 2 Cox regression analysis for factors associated with 1-year mortality

\begin{tabular}{|c|c|c|c|c|}
\hline Variable & \multicolumn{2}{|c|}{ Univariate analysis } & \multicolumn{2}{|c|}{ Multivariate analysis } \\
\hline Age & $1.03(1.02-1.04)$ & $<0.001$ & $1.02(1.00-1.03)$ & 0.008 \\
\hline RRT & $2.14(1.66-2.77)$ & $<0.001$ & $2.09(1.60-2.73)$ & $<0.001$ \\
\hline Vasopressor & $2.33(1.58-3.43)$ & $<0.001$ & $2.14(1.43-3.20)$ & $<0.001$ \\
\hline SOFA & $1.07(1.04-1.10)$ & $<0.001$ & - & - \\
\hline Low skeletal muscle mass & $1.71(1.17-2.49)$ & 0.006 & $1.47(1.01-2.16)$ & 0.046 \\
\hline Rehabilitation & $0.70(0.54-0.90)$ & 0.005 & $0.65(0.50-0.85)$ & 0.002 \\
\hline
\end{tabular}

OR, odds ratio; Cl, confidential interval; RRT, renal replacement therapy; CCI, Charlson Comorbidity Index; SOFA, Sequential Organ Failure Assessment; SMI, skeletal muscle index.

Table 3 Basal characteristics and clinical outcomes of low skeletal muscle mass patients with and without rehabilitation

\begin{tabular}{|c|c|c|c|}
\hline Variable & Rehabilitation $(\mathrm{N}=215)$ & Non-rehabilitation $(\mathrm{N}=206)$ & $\mathrm{P}$ \\
\hline Male & $148(68.8)$ & $141(68.4)$ & 0.931 \\
\hline BMI $\left(\mathrm{kg} / \mathrm{m}^{2}\right)$ & $20.6 \pm 3.5$ & $21.7 \pm 4.5$ & 0.006 \\
\hline $\mathrm{SMI}\left(\mathrm{cm}^{2} / \mathrm{m}^{2}\right)$ & $33.1 \pm 10.1$ & $33.7 \pm 10.2$ & 0.588 \\
\hline SOFA & $8.3 \pm 3.8$ & $8.2 \pm 4.2$ & 0.790 \\
\hline $\mathrm{CCl}$ & $4.4 \pm 2.4$ & $4.2 \pm 2.2$ & 0.435 \\
\hline Multiple vasopressor use & $177(82.3)$ & $162(78.6)$ & 0.340 \\
\hline Renal replacement therapy & $70(32.6)$ & $56(27.2)$ & 0.229 \\
\hline Respiratory & $187(87.0)$ & $186(90.3)$ & 0.285 \\
\hline Abdominal & $15(7.0)$ & $15(7.3)$ & 0.792 \\
\hline Urinary tract & $33(15.3)$ & $16(7.8)$ & 0.347 \\
\hline Soft tissue & $10(4.7)$ & $10(4.9)$ & 0.243 \\
\hline Ventilator day & $24.3 \pm 32.4$ & $28.1 \pm 48.1$ & 0.377 \\
\hline Hospital stay & $73.2 \pm 73.9$ & $35.5 \pm 41.0$ & $<0.001$ \\
\hline ICU stay & $22.5 \pm 25.9$ & $15.9 \pm 21.0$ & 0.004 \\
\hline In-hospital mortality & $56(26.0)$ & $82(39.8)$ & 0.003 \\
\hline
\end{tabular}

Values are expressed as mean \pm standard deviation or $\mathrm{n}(\%)$. BMI, body mass index; SMI, Skeletal muscle index; APACHE II, Acute Physiology and Chronic Health 415 Evaluation II; SOFA, Sequential Organ Failure Assessment; CCI, Charlson Comorbidity Index. 
Table 4 Cox regression analysis for factors associated with 1-year mortality in low skeletal muscle mass

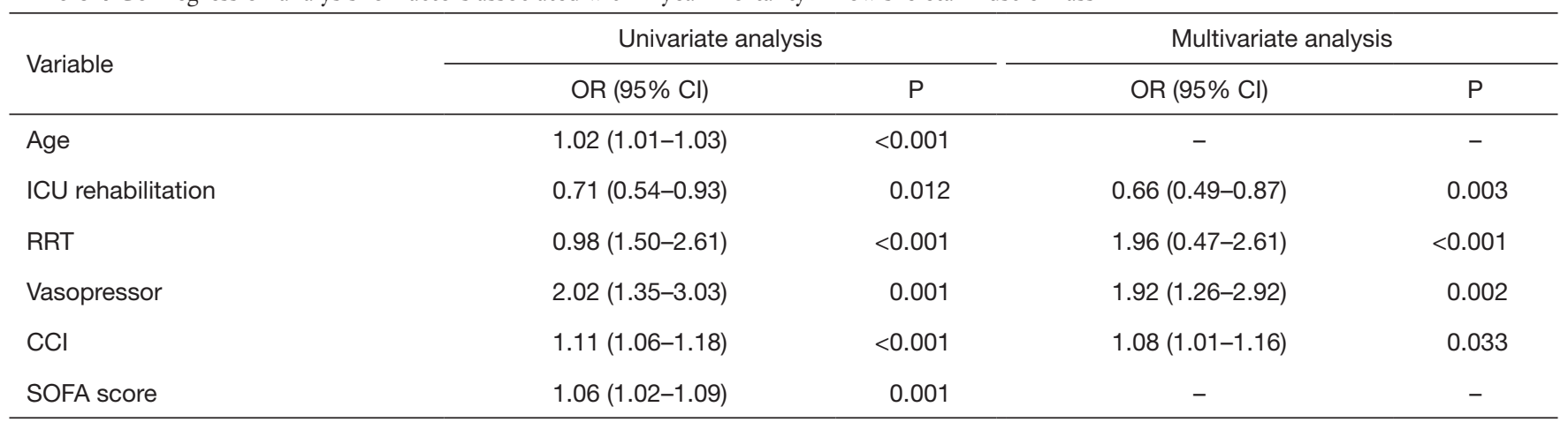

OR, odds ratio; Cl, confidential interval; RRT, renal replacement therapy; CCI, Charlson Comorbidity Index; SOFA, Sequential Organ Failure Assessment.

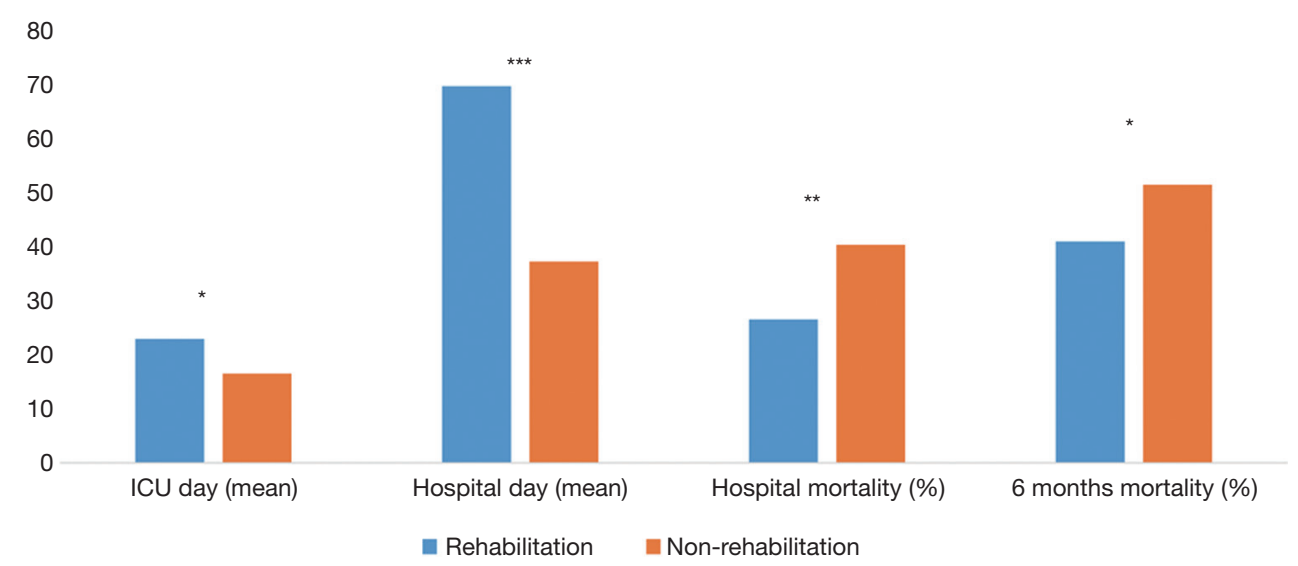

Figure 2 Clinical outcomes in low skeletal muscle mass patients after propensity score matching. After propensity score matching, the mean hospital (69.6 vs. 37.2 days, $\mathrm{P}<0.001)$ and ICU length-of-stay (22.9 vs. 16.5 days, $\mathrm{P}=0.013$ ) were longer in the rehabilitation group. However, hospital mortality $(26.5 \%$ vs. $40.3 \%, \mathrm{P}=0.005)$ and 6-month mortality $(40.9 \%$ vs. $51.4 \%, \mathrm{P}=0.045)$ were lower in the rehabilitation group. *, $\mathrm{P}<0.05 ;{ }^{* *}, \mathrm{P}<0.01 ;{ }^{* * *}, \mathrm{P}<0.001$.

length-of-stay (69.6 vs. 37.2 days, $\mathrm{P}<0.001)$ and ICU length-of-stay (22.9 vs. 16.5 days, $\mathrm{P}=0.013$ ) were longer in the rehabilitation group. Mean ventilator duration did not differ significantly between the two groups. However, hospital mortality ( $26.5 \%$ vs. $40.3 \%, \mathrm{P}=0.005)$ and 6-month mortality $(40.9 \%$ vs. $51.4 \%, \mathrm{P}=0.045)$ were lower in the rehabilitation group. The rate of discharge to home was higher in the rehabilitation group ( $40.3 \%$ vs. $35.4 \%$, $\mathrm{P}=0.016)$. Otherwise, 1 -year mortality did not differ significantly between two groups.

\section{Discussion}

In this study, we analyzed the impact of low skeletal muscle mass and rehabilitation on 1-year mortality in patients with sepsis. Both low skeletal muscle mass and ICU-rehabilitation were important factors for long term prognosis in these patients. Interestingly, ICU-rehabilitation was independently associated with reduced 1-year mortality in septic patients with low skeletal muscle mass. Therefore, ICU-rehabilitation might improve long-term outcomes by enhancing the recovery of low skeletal muscle mass patients with sepsis.

Low skeletal muscle mass is frequently seen in critically ill patients, and it has an adverse impact on clinical outcomes, including a higher mortality, a longer ventilator duration, increased hospital length-of-stay, and delirium (18-21). Previous studies have mainly focused on the impact 
of low skeletal muscle mass on short-term outcomes of sepsis $(5,6)$. In this study, $81.6 \%$ of patients with sepsis had low skeletal muscle mass at admission. Baseline low skeletal muscle mass was associated with medically or physically poor condition at the time of discharge and higher longterm mortality. Considering the high prevalence of low skeletal muscle mass and its prognostic impact on longterm mortality in sepsis, assessing the patient's muscle mass is clinically important. The early screening of low skeletal muscle mass patients facilitates a multidisciplinary treatment approach for high-risk patients (including active nutrition and rehabilitation strategies) and consequently may improve prognosis.

Generally, exercise interventions are known to benefit muscle strength and physical function. However, much remains unclear about whether this same beneficial effect occurs in low skeletal muscle mass (11). In this study, ICUrehabilitation was independently associated with reduced 1-year mortality in septic patients with low skeletal muscle mass, but not in septic patients without low skeletal muscle mass (Figure S1; Tables S1,S2). Unfortunately, this study cannot clearly reveal the mechanism underlying this difference. Presumably, ICU-rehabilitation not only improves strength and function of the muscles as a result of physical activity but also decreases inflammation and oxidative stress. As such, it may prevent or reverse physical impairments and muscle wasting (22-24). These effects of rehabilitation may be more prominent in low skeletal muscle mass patients who are relatively more vulnerable. Further research is required to clarify the mechanisms.

This study has several limitations. First, as a retrospective single-center study, it may have been vulnerable to selection bias because patients without abdominal CT scans were excluded. However, there were no significant differences in clinical outcomes between included patients $(n=516)$ and excluded patients $(\mathrm{n}=396)$, except for early death $(\leq 48 \mathrm{~h}$, $\mathrm{n}=28$, Table S3). Secondly, low skeletal muscle mass was solely diagnosed based on CT imaging, and no functional assessments, such as muscle strength, were performed. In addition, we did not check functional outcomes throughout the follow-up period or at discharge. Instead, we presented a rate of home discharge as an indirect index of function this study. Therefore, this study does not provide enough information on the effect of rehabilitation on functional status and muscle power. This study does not reveal how rehabilitation affects clinical outcomes. Despite these limitations, this study has strong points and is worthwhile. Data show the prognostic relationship between low skeletal muscle mass and sepsis, and the clinical efficacy of early rehabilitation in this population. Also, it is meaningful to identify specific populations in which rehabilitation is effective, to more efficiently use ICU resources. The generalization of rehabilitation for patients in the ICU is challenging due to the high burden of healthcare resources, and concerns about the safety and efficacy of ICUrehabilitation. Patients who are low skeletal muscle mass at admission are more vulnerable to muscle wasting during ICU care compared to those who are not. Selective and intensive rehabilitation for specific patients may lead to cost saving and efficient use of ICU resources. Death from sepsis might be influenced not only by disease severity and early detection and management but also by ICU-rehabilitation. Therefore, clinicians should increase the attention paid to ICU-rehabilitation in patients with sepsis, especially low skeletal muscle mass patients.

\section{Conclusions}

ICU-rehabilitation is important towards improving longterm outcomes in patients with sepsis. In particular, low skeletal muscle mass patients might benefit more from ICU-rehabilitation compared with non-low skeletal muscle mass patients.

\section{Acknowledgments}

None.

\section{Footnote}

Conflicts of Interest: The authors have no conflicts of interest to declare.

Ethical Statement: The study protocol was approved by the institutional review boards of Pusan National University Yangsan Hospital (No. 05-2018-082). The need for informed consent was waived due to the retrospective design of the study. The authors are accountable for all aspects of the work in ensuring that questions related to the accuracy or integrity of any part of the work are appropriately investigated and resolved.

\section{References}

1. Rhee C, Dantes R, Epstein L, et al. Incidence and Trends 
of Sepsis in US Hospitals Using Clinical vs Claims Data, 2009-2014. JAMA 2017;318:1241-9.

2. Perner A, Haase N, Winkel P, et al. Long-term outcomes in patients with severe sepsis randomised to resuscitation with hydroxyethyl starch 130/0.42 or Ringer's acetate. Intensive Care Med 2014;40:927-34.

3. Yende S, Austin S, Rhodes A, et al. Long-Term Quality of Life Among Survivors of Severe Sepsis: Analyses of Two International Trials. Crit Care Med 2016;44:1461-7.

4. Iwashyna TJ, Ely EW, Smith DM, et al. Long-term cognitive impairment and functional disability among survivors of severe sepsis. JAMA 2010;304:1787-94.

5. Shibahashi K, Sugiyama K, Kashiura M, et al. Decreasing skeletal muscle as a risk factor for mortality in elderly patients with sepsis: a retrospective cohort study. J Intensive Care 2017;5:8.

6. Lee Y, Park HK, Kim WY, et al. Muscle Mass Depletion Associated with Poor Outcome of Sepsis in the Emergency Department. Ann Nutr Metab 2018;72:336-44.

7. Mofarrahi M, Sigala I, Guo Y, et al. Autophagy and skeletal muscles in sepsis. PloS One 2012;7:e47265.

8. Schefold JC, Bierbrauer J, Weber-Carstens S. Intensive care unit-acquired weakness (ICUAW) and muscle wasting in critically ill patients with severe sepsis and septic shock. J Cachexia Sarcopenia Muscle 2010;1:147-57.

9. Hashem MD, Nelliot A, Needham DM. Early Mobilization and Rehabilitation in the ICU: Moving Back to the Future. Respir Care 2016;61:971-9.

10. Saitoh M, Ishida J, Doehner W, et al. Sarcopenia, cachexia, and muscle performance in heart failure: Review update 2016. Int J Cardiol 2017;238:5-11.

11. Arai H, Wakabayashi H, Yoshimura Y, et al. Chapter 4 Treatment of sarcopenia. Geriatr Gerontol Int 2018;18 Suppl 1:28-44.

12. Girard TD, Alhazzani W, Kress JP, et al. An Official American Thoracic Society/American College of Chest Physicians Clinical Practice Guideline: Liberation from Mechanical Ventilation in Critically Ill Adults. Rehabilitation Protocols, Ventilator Liberation Protocols, and Cuff Leak Tests. Am J Respir Crit Care Med 2017;195:120-33.

Cite this article as: Kim T, Huh S, Kim SY, Han J, Lee SE, Cho WH, Kim YS, Jeon DS, Yeo HJ. ICU rehabilitation is associated with reduced long-term mortality from sepsis in patients with low skeletal muscle mass: a case control study. Ann Transl Med 2019;7(18):430. doi: 10.21037/atm.2019.08.117
13. Taito S, Taito M, Banno M, et al. Rehabilitation for patients with sepsis: A systematic review and meta-analysis. PloS One 2018;13:e0201292.

14. Tipping CJ, Harrold M, Holland A, et al. The effects of active mobilisation and rehabilitation in ICU on mortality and function: a systematic review. Intensive Care Med 2017;43:171-83.

15. Jeon YK, Shin MJ, Kim CM, et al. Effect of Squat Exercises on Lung Function in Elderly Women with Sarcopenia. J Clin Med 2018. doi: 10.3390/jcm7070167.

16. Singer M, Deutschman CS, Seymour CW, et al. The Third International Consensus Definitions for Sepsis and Septic Shock (Sepsis-3). JAMA 2016;315:801-10.

17. Fearon K, Strasser F, Anker SD, et al. Definition and classification of cancer cachexia: an international consensus. Lancet Oncol 2011;12:489-95.

18. Moisey LL, Mourtzakis M, Cotton BA, et al. Skeletal muscle predicts ventilator-free days, ICU-free days, and mortality in elderly ICU patients. Crit Care 2013;17:R206.

19. Sheean PM, Peterson SJ, Gomez Perez S, et al. The prevalence of sarcopenia in patients with respiratory failure classified as normally nourished using computed tomography and subjective global assessment. JPEN J Parenter Enteral Nutr 2014;38:873-9.

20. Weijs PJ, Looijaard WG, Dekker IM, et al. Low skeletal muscle area is a risk factor for mortality in mechanically ventilated critically ill patients. Crit Care 2014;18:R12.

21. Bellelli G, Zambon A, Volpato S, et al. The association between delirium and sarcopenia in older adult patients admitted to acute geriatrics units: Results from the GLISTEN multicenter observational study. Clin Nutr 2018;37:1498-504.

22. Saggini R, Carmignano SM, Cosenza L, et al. Rehabilitation in Sarcopenic Elderly. Frailty and Sarcopenia - Onset, Development and Clinical Challenges. 2017. DOI: 10.5772/intechopen.69638.

23. Fatouros IG, Jamurtas AZ, Villiotou V, et al. Oxidative stress responses in older men during endurance training and detraining. Med Sci Sports Exerc 2004;36:2065-72.

24. Kizilarslanoglu MC, Kuyumcu ME, Yesil Y, et al. Sarcopenia in critically ill patients. J Anesth 2016;30:884-90. 


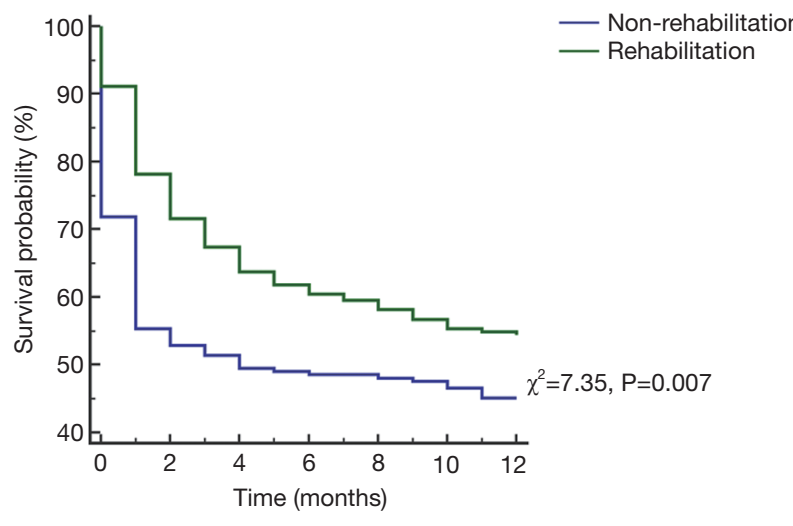

Figure S1 Kaplan Meier analysis for 1-year mortality according to rehabilitation in low skeletal muscle mass patients. Low skeletal muscle mass patients who did not receive ICU rehabilitation had a significantly higher 1-year mortality $\left(\chi^{2}=7.35, \mathrm{P}=0.007\right)$.

Table S1 Basal characteristics and clinical outcomes of non-low skeletal muscle mass patients with and without rehabilitation

\begin{tabular}{|c|c|c|c|}
\hline Variable & Rehabilitation (N=52) & Non-rehabilitation ( $\mathrm{N}=43$ ) & $\mathrm{P}$ \\
\hline Age, years & $64.9 \pm 14.7$ & $64.8 \pm 14.1$ & 0.998 \\
\hline Male & $26(50.0)$ & $28(65.1)$ & 0.1319 \\
\hline BMI $\left(\mathrm{kg} / \mathrm{m}^{2}\right)$ & $23.9 \pm 3.2$ & $23.4 \pm 3.5$ & 0.459 \\
\hline $\mathrm{SMI}\left(\mathrm{cm}^{2} / \mathrm{m}^{2}\right)$ & $54.6 \pm 12.6$ & $61.1 \pm 17.2$ & 0.036 \\
\hline APACHE II & $15.5 \pm 6.3$ & $14.5 \pm 7.3$ & 0.507 \\
\hline SOFA & $8.5 \pm 4.6$ & $7.9 \pm 4.2$ & 0.523 \\
\hline $\mathrm{CCl}$ & $4.2 \pm 2.3$ & $4.4 \pm 2.8$ & 0.717 \\
\hline Multiple vasopressor use & $38(73.1)$ & $33(76.7)$ & 0.682 \\
\hline Renal replacement therapy & $18(34.6)$ & $10(23.3)$ & 0.227 \\
\hline Lactate on admission & $2.7 \pm 4.5$ & $7.1 \pm 27.9$ & 0.315 \\
\hline \multicolumn{4}{|l|}{ Infection source } \\
\hline Respiratory & $46(88.5)$ & $35(81.4)$ & 0.333 \\
\hline Abdominal & $5(9.6)$ & $4(9.3)$ & 0.361 \\
\hline Urinary tract & $11(21.2)$ & $3(7.0)$ & 0.025 \\
\hline Soft tissue & $5(9.6)$ & $3(7.0)$ & \\
\hline Ventilator day & $34.8 \pm 40.0$ & $14.7 \pm 13.8$ & 0.002 \\
\hline Hospital stay & $60.5 \pm 53.5$ & $25.2 \pm 21.3$ & $<0.001$ \\
\hline ICU stay & $20.9 \pm 29.2$ & $13.3 \pm 21.3$ & 0.119 \\
\hline In-hospital mortality & $10(19.2)$ & $14(32.6)$ & 0.137 \\
\hline Discharge to home & $33(63.5)$ & 23 (53.5) & 0.325 \\
\hline 6-month mortality & $11(21.2)$ & $14(32.6)$ & 0.209 \\
\hline 1-year mortality & 15 (28.8) & $16(37.2)$ & 0.387 \\
\hline
\end{tabular}

Values are expressed as mean \pm standard deviation or $\mathrm{n}(\%)$. BMI, body mass index; SMI, skeletal muscle index; APACHE II, Acute Physiology and Chronic Health Evaluation II; SOFA, Sequential Organ Failure Assessment; CCI, Charlson Comorbidity Index.

Table S2 Cox regression analysis for factors associated with 1-year mortality in non-low skeletal muscle mass patients

\begin{tabular}{|c|c|c|c|c|}
\hline \multirow{2}{*}{ Variable } & \multicolumn{2}{|c|}{ Univariate analysis } & \multicolumn{2}{|c|}{ Multivariate analysis } \\
\hline & OR (95\% Cl) & $P$ & OR $(95 \% \mathrm{Cl})$ & $P$ \\
\hline Age & $1.06(1.03-1.10)$ & $<0.001$ & $1.06(1.03-1.09)$ & $<0.001$ \\
\hline ICU rehabilitation & $0.68(0.34-1.38)$ & 0.290 & - & - \\
\hline RRT & $3.42(1.68-6.96)$ & 0.001 & $2.60(1.24-5.47)$ & 0.011 \\
\hline Vasopressor & $5.86(1.40-24.56)$ & 0.016 & - & - \\
\hline $\mathrm{CCl}$ & $1.33(1.15-1.53)$ & $<0.001$ & - & - \\
\hline SOFA & $1.13(1.05-1.23)$ & 0.002 & - & - \\
\hline
\end{tabular}

ICU, intensive care unit; RRT, renal replacement therapy; CCI, Charlson Comorbidity Index; SOFA: Sequential Organ Failure Assessment.

Table S3 Outcomes of included patient and excluded patients, except early death ( $\leq 48$ hours)

\begin{tabular}{lccc}
\hline Variable & Included patients $(\mathrm{n}=516)(\%)$ & Excluded patients $(\mathrm{n}=396)(\%)$ & $\mathrm{P}$ \\
\hline Hospital mortality & $167(32.4)$ & $134(33.8)$ & 0.639 \\
6-month mortality & $168(32.6)$ & $135(34.1)$ & 0.626 \\
1-year mortality & $174(33.7)$ & $135(34.1)$ & 0.907 \\
\hline
\end{tabular}

There were no significant differences in hospital mortality, 6-month mortality, and 1-year mortality between included patients $(n=516)$ and excluded patients $(n=396)$, except early death ( $\leq 48$ hours, $n=28)$. 